\title{
Vibration Suppression and Angle Tracking of a Fire-Rescue Ladder
}

\author{
Jiali Feng - Xiuyu He* - Shuang Zhang - Guang Li
}

Received: date / Accepted: date

\begin{abstract}
This paper mainly considers vibration suppression and angle tracking of a fire-rescue ladder system. The dynamical model is regarded as a segmented Euler-Bernoulli beam with gravity and tip mass, described by a set of motion equations and boundary conditions. Based on the nonlinear Euler-Bernoulli beam model, two active boundary controllers are proposed to achieve the control objectives. The elastic deflection and the angular error in the closed-loop system are proven to converge exponentially to a small neighborhood of zero. Numerical simulations based on finite difference method verify the effectiveness and the ascendancy of active boundary controllers.
\end{abstract}

Keywords Vibration Suppression · Angle Tracking · Active Boundary Control · Nonuniform Euler-Bernoulli Beam · Distributed Parameter System

\section{Introduction}

Modern fire-rescue ladders are becoming more and more lightweight for increasing outreach and improving operational velocity. For example the ladder shown in Fig. 1 is over 53 meters long and weighs over 4800 kilograms. The cage that is installed on the top of the ladder

J. Feng · S. Zhang are with School of Automation and Electrical Engineering and Institute of Artificial Intelligence, University of Science and Technology Beijing, Beijing 100083, China.

$\mathrm{X}$. He is with School of Automation and Electrical Engineering and Institute of Artificial Intelligence, University of Science and Technology Beijing, Beijing 100083, China, and also with Shunde Graduate School, University of Science and Technology Beijing, Foshan 528399, China.

G. Li is with School of Engineering and Materials Science, Queen Mary University of London, Mile End Road, London E1 4Ns, UK.

E-mail: xiuyuhe@ieee.org weighs over 50 kilograms, and it can take on board up to 225 kilograms of load[32]. Indeed, the flexible fire-rescue ladder is a kind of large-scale flexible manipulator that should be considered as an example of distributed parameter systems (DPS). As a result of using light material, the stiffness of the fire-rescue ladder decreases and the ladder becomes more susceptible to vibration problem caused by wind, motion and so on.

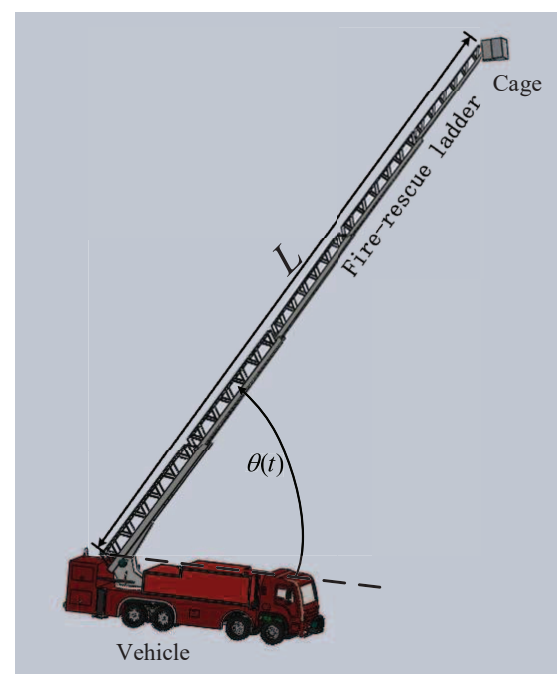

Fig. 1 A fire-rescue ladder.

The problems of vibration suppression and target tracking have been considered in many DPS, e.g. wave equation systems[12], [23], heat equation systems[21], [37], string systems[28], beam systems[22], [38], [11], [9], and so on. The stabilization problem of a one-dimensional wave equation is considered in [23] and a controller together with an observer is designed using the backstepping method. The stabilization of a system that 
is described by a heat equation is considered in [37] and a boundary control law is advocated. By using a Schrödinger equation, the closed-loop heat equation system is proven to have exponential stability with the Riesz basis property. In [40], a boundary control strategy is proposed to reduce the vibration of string systems with input backlash. A crane with varying cable length is considered in [36], and a tracking method is advocate. In [9], a force control strategy is used for a one-link flexible arm that is modeled by a Timoshenko beam. Comparing with the Timoshenko beam model which considers both bending and shear deformations of a beam [4], the Euler-Bernoulli beam model is easier, just considering bending deformations [6]. In applications, the Timoshenkon beam is suitable for describing short rods or thick plates [2]; the Euler-Bernoulli beam is suitable for describing long and thin rods or thin plates [11]. A class of linear cascaded system that composed by linear time-invariant systems and Euler-Bernoulli beam systems is considered in [38], and a feedback controller is designed to achieve the exponential stability. In [22], [16], boundary controllers or adaptive boundary controllers are used to suppress vibration of different EulerBernoulli beams. Boundary control is economical and practical because it just uses several sensors and actuators on two ends of a controlled plant [24], and boundary control has wide application, such as marine riser systems [7], [43], robot arms [8], crane systems [20], [17], flapping wing micro aerial vehicles [15], spacecraft systems [26], string systems [41], [42] and aerial refueling hose systems [27]. Therefore, boundary control could be used in fire-rescue ladders.

In reviewing the study of fire-rescue ladders, the models have developed from rigid beam to flexible beam for the purpose of improving accuracy, and the control strategies have made corresponding changes. Two 30-metre fire-rescue ladders are modeled as rigid beams in [34] and [25], and the control strategies for trajectory tracking are based on a decentralized approach. However, the rigid models ignore some infinitedimensional characteristics, and cannot reach a good simulation of oscillation, especially high-frequency oscillation because of the disregard [32]. Because of the fact that the ratio of the length of the ladder to the minimum gyration radius of the cross section is relatively large, the ladder should be modeled as a EulerBernoulli beam. In [45], authors use a piecewise continuous Euler-Bernoulli beam to establish a model for a 53-metre ladder. Comparing homogenized model [1], the piecewise continuous beam model is more suitable for the fire-rescue ladders where the upper sections have smaller cross-sections than the lower sections. But in [45], the controller designed to adjust system modes and to damp vibration is based on a decentralized EulerBernoulli beam model. This decentralized approach also lets the ladder system loss some infinite-dimensional characteristics. In this paper, a set of boundary control laws will be designed based on the flexible EulerBernoulli beam model directly rather than a decentralized Euler-Bernoulli beam model.

Overall, this paper makes contributions to the study of fire-rescue ladders and the expansion of boundary control's applications. The main contribution is that the boundary control strategy is proposed directly based on the partial differential equations model. This approach of control designing avoids the loss of the information of the PDE model. Meanwhile, the closed-loop system is uniform ultimate bounded in theory and numerical simulations verify the effectiveness of the boundary controllers.

\section{Problem Formulation}

As a thin plate, the fire-rescue ladder shown in Fig. 1 is modeled as an Euler-Bernoulli beam (Fig. 2). Different segments are not shown in Fig. 2. $L$ denotes the length after extension. $\theta(t)$ denotes the flexible angular position. $w(z, t)$ is vibration deflection at the position $z$ for time $t$. In addition, we mark $(\cdot)^{\prime}=\partial(\cdot) / \partial z$, $(\cdot)^{\prime \prime}=\partial^{2}(\cdot) / \partial z^{2},(\cdot)^{\prime \prime \prime}=\partial^{3}(\cdot) / \partial z^{3},(\cdot)^{\prime \prime \prime \prime}=\partial^{4}(\cdot) / \partial z^{4}$, $(\cdot)=\partial(\cdot) / \partial t$ and $(\cdot)=\partial^{2}(\cdot) / \partial t^{2}$.

\subsection{Dynamical Model}

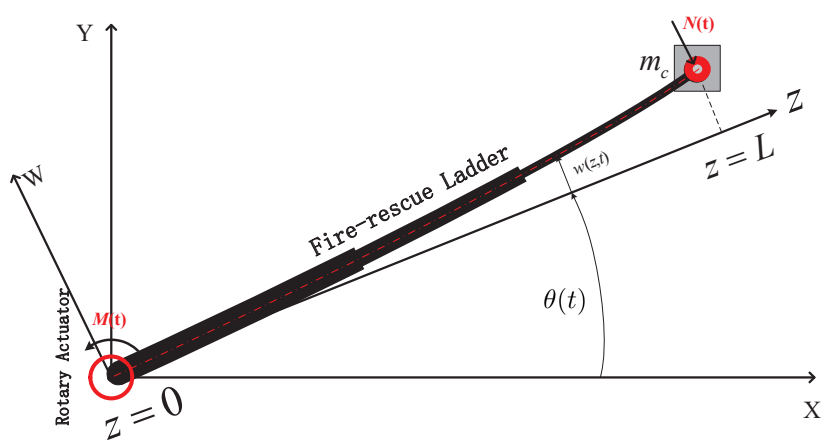

Fig. 2 Dynamical model of the fire-rescue ladder.

The dynamical model of the ladder is divided into $S$ segments. $\aleph_{n}$ means the $n$th segment, where $n=$ $1,2, \cdots, S$, and $z_{n-1}$ and $z_{n}$ are the lower and upper ends of $\aleph_{n}$, respectively. Further, we have $z_{0}=0$, $z_{S}=L, \aleph_{n}=\left[z_{n-1}, z_{n}\right)$. Because every segment on the ladder is considered to be a uniform one, we then have 
$\rho(z)=\rho_{n}$ and $E I(z)=E I_{n}$ where $z \in \aleph_{n}, \rho_{n}$ and $E I_{n}$ denote the linear density and the bending stiffness of $\aleph_{n}$, respectively. Since the ladder tapers from $\aleph_{1}$ to $\aleph_{S}$, we then derive $\rho_{S} \leq \rho_{n+1}<\rho_{n} \leq \rho_{1}$ and $E I_{S} \leq E I_{n+1}<E I_{n} \leq E I_{1}$.

Moreover, this paper mainly considers that the deformation of the ladder is along the central axis. The connection between the central axes of two adjacen$\mathrm{t}$ sections is smooth. Therefore, at the boundary of a segment $z_{n}$, the deflection of the ladder is continuous, namely $w\left(z_{n}^{-}, t\right)=w\left(z_{n}^{+}, t\right)$ and the slope of the deflection is also continuous, namely $w^{\prime}\left(z_{n}^{-}, t\right)=w^{\prime}\left(z_{n}^{+}, t\right) . z_{n}^{-}$ and $z_{n}^{+}$belong to segments $\aleph_{n}$ and $\aleph_{n+1}$, respectively, and $z_{n}^{-}=z_{n}=z_{n}^{+}$. Defining $P(z, t)=w(z, t)+z \theta(t)$, we further have $P\left(z_{n}^{-}, t\right)=P\left(z_{n}^{+}, t\right)$ and $P^{\prime}\left(z_{n}^{-}, t\right)=$ $P^{\prime}\left(z_{n}^{+}, t\right)$.

The kinetic energy and the potential energy are similar with [32], and the virtual work $\delta W_{n c}$ done by the non-conservative force is consist of the virtual work done by damping and the boundary controllers.

$$
\begin{aligned}
\delta W_{n c}(t)= & M(t) \delta \theta(t)+N(t) \delta P(L, t) \\
& -\sum_{n=1}^{S} \int_{z_{n-1}^{+}}^{z_{n}^{-}} c \dot{P}(z, t) \delta P(z, t) d z,
\end{aligned}
$$

where $c$ describes the damping coefficient of the ladder.

According to Hamilton's principle and using the conditions $P\left(z_{n}^{-}, t\right)=P\left(z_{n}^{+}, t\right)$ and $P^{\prime}\left(z_{n}^{-}, t\right)=P^{\prime}\left(z_{n}^{+}, t\right)$, the dynamical model of the ladder can be obtained. Equations of motion are

$$
\begin{aligned}
& \rho_{n}[\ddot{P}(z, t)+g \cos \theta(t)]+E I_{n} P^{\prime \prime \prime \prime}(z, t)+c \dot{P}(z, t)=0, \\
& -\sum_{n=1}^{S} g \rho_{n} \int_{z_{n-1}^{+}}^{z_{n}^{-}} w(z, t) \sin \theta(t) d z+J_{A} \ddot{\theta}(t) \\
& \quad-E I_{1} P^{\prime \prime}(0, t)-m_{c} g w(L, t) \sin \theta(t)=M(t),(3)
\end{aligned}
$$

where $z \in \aleph_{n}$, and $J_{A}$ describes the moment of inertia of the rotary actuator. Boundary conditions are

$$
\begin{aligned}
& P(0, t)=0, \\
& P^{\prime}(0, t)=\theta(t), \\
& P^{\prime \prime}(L, t)=0, \\
& E I_{n} P^{\prime \prime}\left(z_{n}^{-}, t\right)=E I_{n+1} P^{\prime \prime}\left(z_{n}^{+}, t\right), \\
& E I_{n} P^{\prime \prime \prime}\left(z_{n}^{-}, t\right)=E I_{n+1} P^{\prime \prime \prime}\left(z_{n}^{+}, t\right), \\
& m_{c}[\ddot{P}(L, t)+g \cos \theta(t)]-E I_{S} P^{\prime \prime \prime}(L, t)=N(t) .
\end{aligned}
$$

Remark 1 Using the definition of $P(z, t), P(z, t)=w(z, t)$ $+z \theta(t)$, we have $P(0, t)=w(0, t), w^{\prime \prime}(z, t)=P^{\prime \prime}(z, t)$ and $w^{\prime \prime \prime}(z, t)=P^{\prime \prime \prime}(z, t)$. Meanwhile, on the basis of boundary conditions, we have $w(0, t)=0, w^{\prime}(0, t)=$ $P^{\prime}(0, t)-\theta(t)=0$ and $w^{\prime \prime}(L, t)=0$.

\subsection{Preliminaries}

This subsection mainly provides some frequently used inequations for the following contents. Combining the Remark 1 and the Young's inequality [33], we have

$$
\begin{aligned}
& \sum_{n=1}^{S} \int_{z_{n-1}^{+}}^{z_{n}^{-}}[w(z, t)]^{2} d z \leq 2 L \sum_{n=1}^{S} \int_{z_{n-1}^{+}}^{z_{n}^{-}}\left[w^{\prime}(z, t)\right]^{2} d z \\
& \sum_{n=1}^{S} \int_{z_{n-1}^{+}}^{z_{n}^{-}}\left[w^{\prime}(z, t)\right]^{2} d z \leq 2 L \sum_{n=1}^{S} \int_{z_{n-1}^{+}}^{z_{n}^{-}}\left[w^{\prime \prime}(z, t)\right]^{2} d z,
\end{aligned}
$$

For any point $z_{l}$ along the ladder, we further have

$w\left(z_{l}, t\right)^{2} \leq \sum_{n=1}^{S} \int_{z_{n-1}^{+}}^{z_{n}^{-}} 4\left[w^{\prime}(z, t)\right]^{2} d z$.

\section{Control Design}

The control objectives in this paper include suppressing the vibration to a small scale and tracking the target angle.

\subsection{Boundary Control Strategy}

By using Lyapunov's direct method, two boundary controllers are presented as follows.

$$
\begin{aligned}
M(t)= & -E I_{1} P^{\prime \prime}(0, t)-m_{c} g w(L, t) \sin \theta(t)-J_{A} \dot{\theta}(t) \\
& -\frac{k+2 k_{\theta}}{2 \gamma}\left[\dot{\theta}(t)+\theta(t)-\theta_{d}\right] \\
N(t)= & m_{c} g \cos \theta(t)-\frac{m_{c}(\alpha-\xi L)}{\beta} \dot{w}(L, t)-k_{u} u_{a}(t)
\end{aligned}
$$

where $u_{a}(t)=\frac{1}{\alpha} \dot{P}(L, t)+\frac{(\alpha-\xi L)}{\beta \alpha} w(L, t), \theta_{d}$ is the target angle while $k, k_{u}, k_{\theta}, \alpha, \beta$ and $\xi$ are positive constants. Fig. 3 illustrates the work principle of the close-loop system.

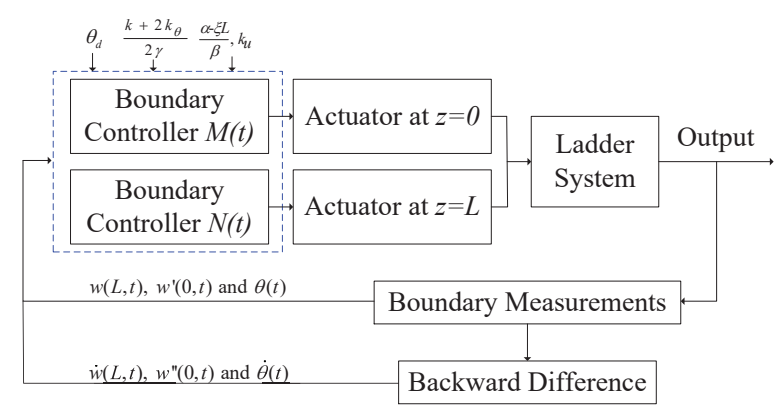

Fig. 3 Block diagram about the process of the closed-loop system. 
Remark 2 The controllers designed in this paper need minimum three sensors: a laser displacement sensor, an inclinometer and an encoder can measure $w(L, t)$, $w^{\prime}(0, t)$ and $\theta(t)$, respectively. Then $\dot{w}(L, t), w^{\prime \prime}(0, t)$ and $\dot{\theta}(t)$ can be calculated by one-order difference of $w(L, t), w^{\prime}(0, t)$ and $\theta(t)$, respectively.

\subsection{Stability Analysis}

For the fire-rescue ladder system described in (2)-(9), the boundary controllers (13) and (14) are designed to damp the vibration and track the target angle. By designing appropriate Lyapunov function candidate, we can prove the efficiency of the controllers [29]. The Lyapunov function candidate in this paper is as follows.

$V(t)=V_{I}(t)+V_{I I}(t)+V_{I I I}(t)$

where

$$
\begin{aligned}
V_{I}(t)= & \frac{\beta}{2} \sum_{n=1}^{S} \int_{z_{n-1}^{+}}^{z_{n}^{-}} \rho_{n}[\dot{P}(z, t)]^{2} d z \\
& +\frac{\beta}{2} \sum_{n=1}^{S} \int_{z_{n-1}^{+}}^{z_{n}^{-}} E I_{n}\left[P^{\prime \prime}(z, t)\right]^{2} d z \\
V_{I I}(t)= & \frac{\gamma J_{A}}{2}\left[\dot{\theta}(t)+\theta_{e}(t)\right]^{2}+\frac{k}{2}\left[\theta_{e}(t)\right]^{2}+\frac{\beta \alpha^{2} m_{c}}{2} u_{a}(t)^{2} \\
V_{I I I}(t)= & \alpha \sum_{n=1}^{S} \int_{z_{n-1}^{+}}^{z_{n}^{-}} \rho_{n} w(z, t) \dot{P}(z, t) d z \\
& +\zeta \sum_{n=1}^{S} \int_{z_{n-1}^{+}}^{z_{n}^{-}} \rho_{n}(L-z) w^{\prime}(z, t) \dot{P}(z, t) d z \\
& -\xi \sum_{n=1}^{S} \int_{z_{n-1}^{+}}^{z_{n}^{-}} \rho_{n} z w^{\prime}(z, t) \dot{P}(z, t) d z
\end{aligned}
$$

where $\gamma$ and $\zeta$ are positive constants and $\theta_{e}(t)=\theta(t)-$ $\theta_{d}$.

The Lyapunov function candidate and the closedloop ladder system can be analysed through the following three steps.

Step 1: The Lyapunov function candidate defined by (15) has upper and lower boundaries, namely, $0 \leq \lambda_{1}$ $\times\left(V_{I}(t)+V_{I I}(t)\right) \leq V(t) \leq \lambda_{2}\left(V_{I}(t)+V_{I I}(t)\right)$, where $\lambda_{1}$ and $\lambda_{2}$ are positive constants.

Proof : See Appendix A.

Step 2: The first derivative of Lyapunov function candidate given by $(15)$ satisfies $\dot{V}(t)<-\lambda V(t)+\epsilon$, where $\lambda$ is a positive constant.

Proof : See Appendix B.

Step 3: Following consequences exist in the fire-rescue ladder system: a) The flexible ladder's elastic deflection $w(z, t)$ can stay in the compact set $\Omega_{1 w}$ and eventually converge to the compact set $\Omega_{2 w}$.

$$
\begin{aligned}
& \Omega_{1 w}:=\left\{w(z, t) \in \mathbb{R}|| w(z, t) \mid \leq D_{1}\right\}, \\
& \Omega_{2 w}:=\left\{w(z, t) \in \mathbb{R}\left|\lim _{t \rightarrow \infty}\right| w(z, t) \mid \leq D_{2}\right\}, \\
& \forall(z, t) \in\left[z_{n-1}^{+}, z_{n}^{-}\right) \times[0, \infty), n \in\{1,2, \ldots, S\},
\end{aligned}
$$

where $D_{1}=\sqrt{\frac{16 L}{\lambda_{1} \beta E I_{n}}\left[V(0)+\frac{\varepsilon}{\lambda}\right]}$ and $D_{2}=\sqrt{\frac{16 L \varepsilon}{\lambda \lambda_{1} \beta E I_{n}}}$.

b) The flexible ladder's angular error $\theta_{e}(t)=\theta(t)-\theta_{d}$ can stay in the compact set $\Omega_{1 \theta}$ and eventually converge to the compact set $\Omega_{2 \theta}$.

$\Omega_{1 \theta}:=\left\{\theta_{e}(t) \in R|| \theta_{e}(t) \mid \leq D_{3}\right\}$,

$\Omega_{2 \theta}:=\left\{\theta_{e}(t) \in R\left|\lim _{t \rightarrow \infty}\right| \theta_{e}(t) \mid \leq D_{4}\right\}, \quad \forall t \in[0, \infty)$,

where $D_{3}=\sqrt{\frac{2}{k \lambda_{1}}\left[V(0)+\frac{\varepsilon}{\lambda}\right]}$ and $D_{4}=\sqrt{\frac{2 \varepsilon}{k \lambda \lambda_{1}}}$.

Proof : See Appendix C.

Therefore, for the system described by (2) to (3) and boundary condition (4) to (9), under the control laws (13) and (14), we can conclude that the closedloop ladder system is ultimately uniformly bounded.

Remark $3 V(t)$ can converge to constant $\frac{\varepsilon}{\lambda}$. Combining the Step 1, we have $V_{I}(t)+V_{I I}(t)$ can also converge to constant $\frac{\varepsilon}{\lambda \lambda_{1}}$. Since $V_{I}(t)$ and $V_{I I}(t)$ both can be described as a sum of squares (16)-(17), namely, $V_{I}(t) \geq 0$ and $V_{I I}(t) \geq 0, V_{I}(t)$ and $V_{I I}(t)$ are also convergent. And every square term in $V_{I}(t)$ and $V_{I I}(t)$ is convergent, in other words, $\dot{P}(z, t), P^{\prime \prime}(z, t)$ and $u_{a}(t)$ are convergent. Besides, using Step 3, we further have that the potential energy and the kinetic energy are convergent.

\section{Simulation}

To illustrate the flexible fire-rescue ladder system, we set the following initial conditions $[45]: w(z, 0)=-\frac{0.05}{L^{3}} z^{3}-$ $\frac{0.15}{L^{2}} z^{2}$ and $\theta(0)=68^{\circ}$. And the physical parameter$\mathrm{s}$ are $L=53 \mathrm{~m}, m_{c}=275 \mathrm{~kg}, J_{A}=900 \mathrm{~kg} \cdot \mathrm{m}^{2}$, $S=2, c=0.5 \mathrm{~N} \cdot \mathrm{s} / \mathrm{m}, \rho_{n}=\left[\begin{array}{ll}160 & 63\end{array}\right] \mathrm{kg} / \mathrm{m}$ and $E I_{n}=\left[\begin{array}{ll}10.578 & 5.354\end{array}\right] \times 10^{7} \mathrm{~N} / \mathrm{m}$. The target angle $\theta_{d}$ is $73^{\circ}$.

Remark 4 In the numerical simulation, this paper gives some initial conditions and values of physical parameters. Considering the influence of gravity and the cage, the initial value elastic deflection is negative. Because the fire-rescue ladder is always on an extension state and has load in cage in practical application, the ladder's length $L$ in simulations is $53 \mathrm{~m}$; the initial angle of the ladder is $68^{\circ}$ and the weight of the cage $m_{c}$ is $275 \mathrm{~kg}$. 
This section mainly discusses the moving states of the flexible ladder under three different control states: without control, PD control and boundary control. When the flexible ladder moves without control, namely, $M(t)$ and $N(t)$ are equal to zero. Fig. 4 shows the moving situation when the fire-rescue ladder has no control. The ladder moves in an arc because of gravity and damping force. Fig. 5 shows the angle of the fire-rescue ladder without control. From Fig. 5, we can find that the ladder has no trend that the ladder approaches the target position. Fig. 6 and Fig. 12 show the vibration offset of the whole ladder without control and the top of the ladder with three different control states, respectively. From Fig. 6 and the blue dotted line in Fig. 12, we can find that the vibration offset is divergent. With intent to ensure the fire rescue operation go with swing, the flexible ladder has to work under control.

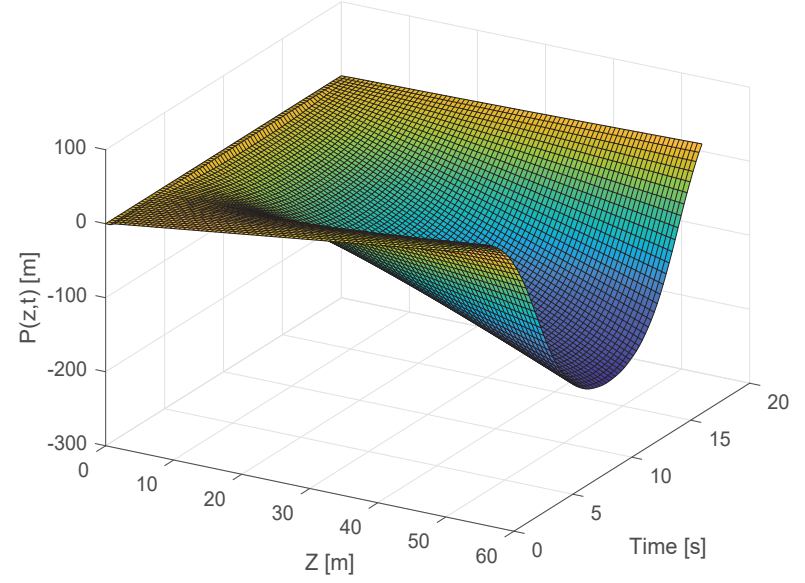

Fig. 4 The overall state of the fire-rescue ladder without control.

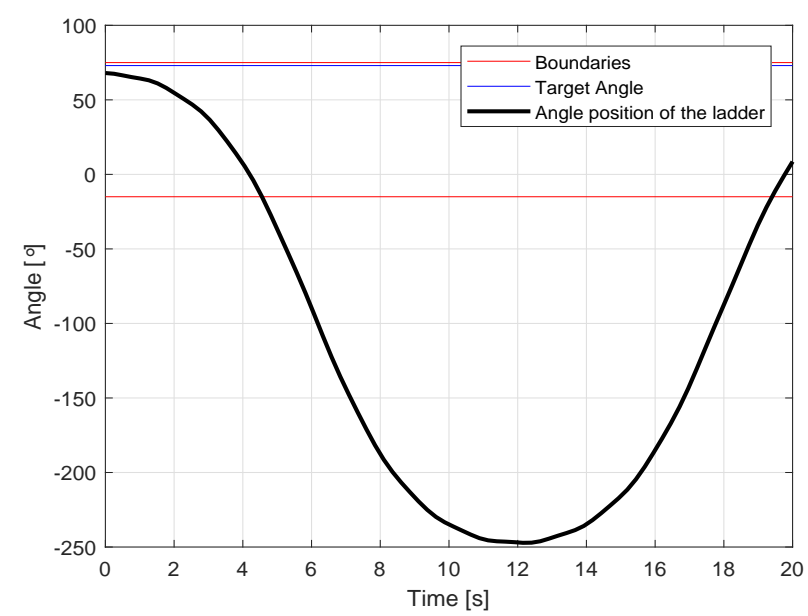

Fig. 5 The angle of the fire-rescue ladder without control.

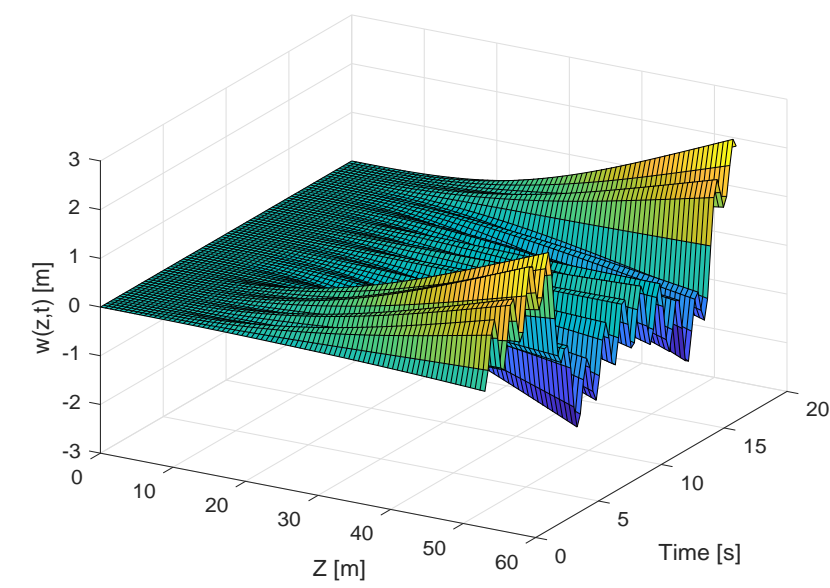

Fig. 6 The vibration offset of the entire ladder without control.

As the comparison, proportional differential (PD) controllers are introduced into the fire-rescue ladder model. The PD controllers are

$\begin{aligned} M_{P D}(t) & =-k_{p \theta}\left[\theta(t)-\theta_{d}\right]-k_{d \theta} \dot{\theta}(t), \\ N_{P D}(t) & =-k_{p w} w(L, t)-k_{d w} \dot{w}(L, t),\end{aligned}$

where the control parameters are $k_{p \theta}=5.7 \times 10^{7}, k_{d \theta}=$ $8.0 \times 10^{6}, k_{p w}=1.0 \times 10^{5}$ and $k_{d w}=6 \times 10^{3}$. Fig. 7 demonstrates the moving situation of the ladder under PD control. Fig. 8 demonstrates the vibration offset of the entire ladder with PD control. From Fig. 8, we can find that the ladder's elastic deflection converges constantly to a stable state and the range of elastic deflection is $4.2 \mathrm{~m}$. The broken green lines in Fig. 11 and Fig. 12 show that the closed-loop ladder system can attain stabilization within $13 \mathrm{~s}$. The angle and the vibration offset can converge within $4 \mathrm{~s}$ and $13 \mathrm{~s}$, respectively. The overshoots of the angle and the vibration offset are $1.4^{\circ}$ and $2.1 \mathrm{~m}$ (Fig. 8), respectively. The overshoot of the vibration offset at the top of the ladder $w(L, t)$ is $0.8 \mathrm{~m}$. Since the flexible ladder works at a relatively large angle, big overshoots may bring about that the ladder exceeds the mechanical limit and even suffers damage.

For the engineers' convenience, the terms $\sin \theta(t)$ and $\cos \theta(t)$ in the controllers (13)-(14) can use Taylor series to approximate. After Taylor series approximation at $60^{\circ}$, the controllers (13)-(14) can be turned into

$$
\begin{aligned}
M_{T}(t)= & -E I_{1} P^{\prime \prime}(0, t)-m_{c} g w(L, t)\left(\frac{\sqrt{3}}{2}+\frac{\theta(t)}{2}\right. \\
& \left.-\frac{\sqrt{3} \theta(t)^{2}}{4}-\frac{\theta(t)^{3}}{12}\right)-J_{A} \dot{\theta}(t) \\
& -\frac{k+2 k_{\theta}}{2 \gamma}\left[\dot{\theta}(t)+\theta(t)-\theta_{d}\right],
\end{aligned}
$$




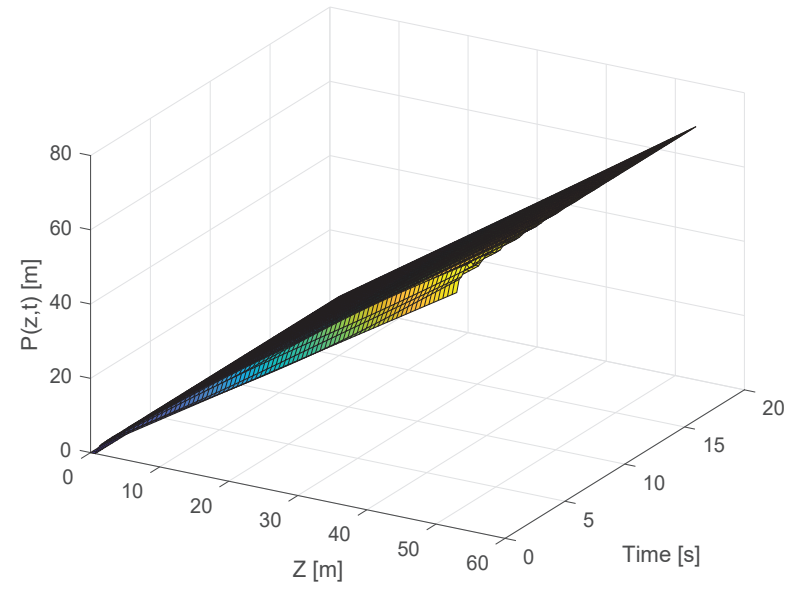

Fig. 7 The overall state of the fire-rescue ladder with PD control.

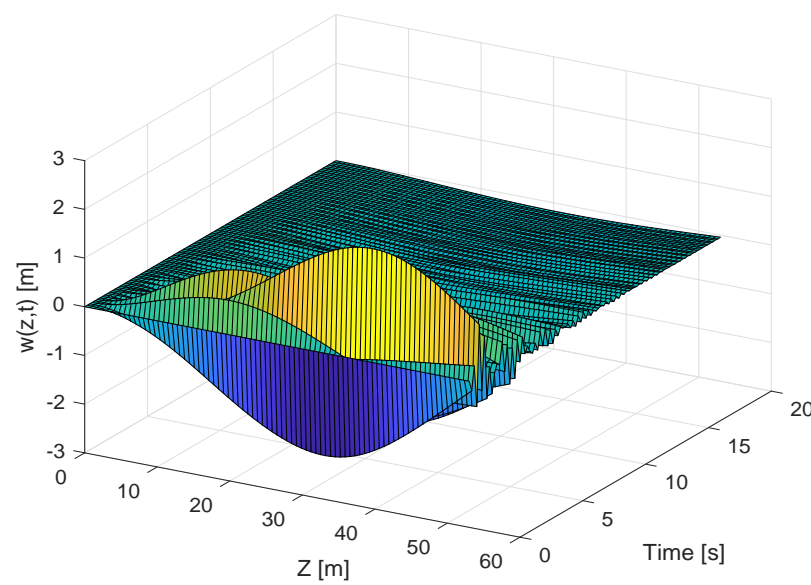

Fig. 8 The vibration offset of the entire ladder with PD control.

$$
\begin{aligned}
N_{T}(t)= & m_{c} g\left(0.5-\frac{\sqrt{3} \theta(t)}{2}-0.25 \theta(t)^{2}+\frac{\sqrt{3} \theta(t)^{3}}{12}\right) \\
& -\frac{m_{c}(\alpha-\xi L)}{\beta} \dot{w}(L, t)-k_{u} u_{a}(t) .
\end{aligned}
$$

Fig. 9-Fig. 12 show the control effect of the ladder with the boundary controllers after Taylor series approximating (21)-(22). The control parameters of the boundary control laws are $\frac{k+2 k_{\theta}}{2 \gamma}=8.02 \times 10^{6}, \alpha=9, \beta=1$, $\xi=0.45$ and $k_{u}=12700$. Fig. 9 demonstrates the moving situation of the ladder under boundary control. Fig. 10 demonstrates the vibration offset of the entire ladder with boundary control. From Fig. 10, we can find that the ladder's elastic deflection converges constantly to a stable state and the range of elastic deflection is 1.8m. The red line in Fig. 11 and Fig. 12 show that the closed-loop ladder system can attain stabilization within $4 \mathrm{~s}$. The angle and the vibration offset can converge within $2 \mathrm{~s}$ and $4 \mathrm{~s}$, respectively. The overshoots of the angle and of the vibration offset are $0.2^{\circ}$ and $1.4 \mathrm{~m}$, respectively. The overshoot of the vibration offset at the top of the ladder $w(L, t)$ is $0.2 \mathrm{~m}$.

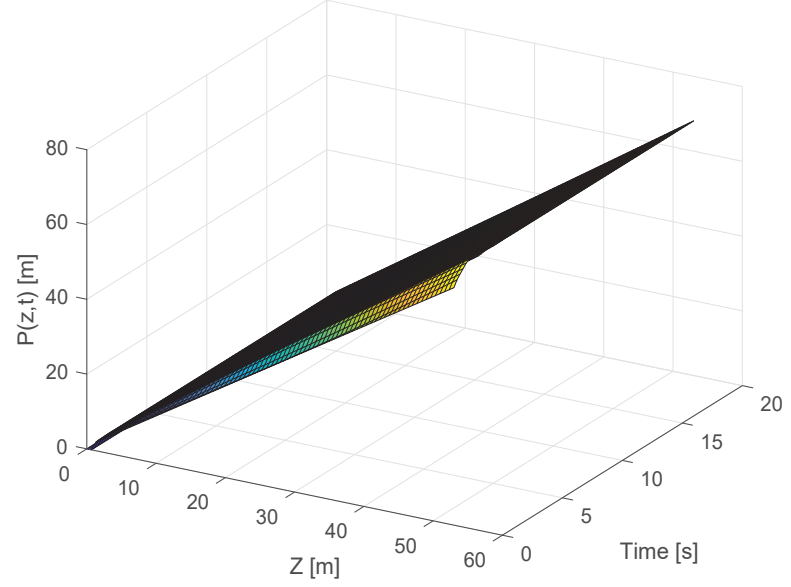

Fig. 9 The overall state of the fire-rescue ladder with boundary control.

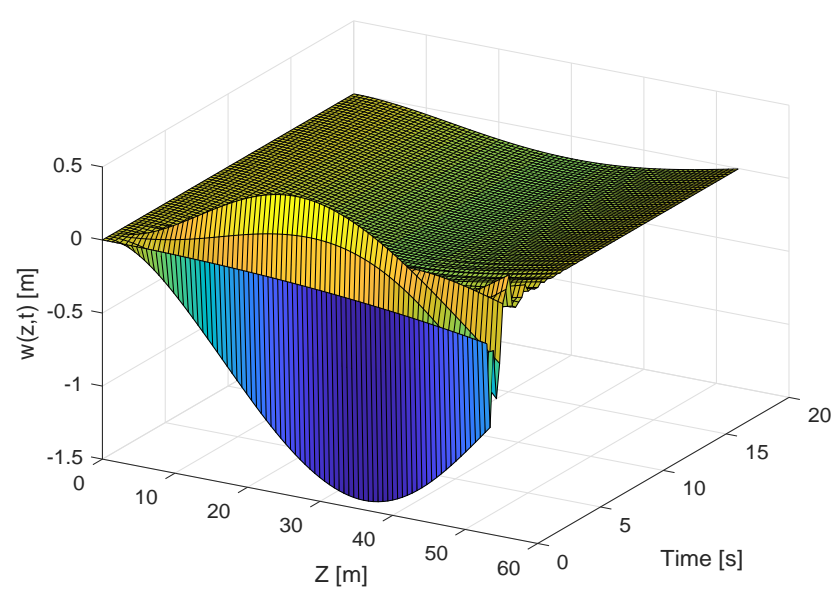

Fig. 10 The vibration offset of the entire fire-rescue ladder with boundary control.

Comparing with the simulation without control, the boundary control laws proposed in this paper have effectiveness, i.e., the boundary control laws can suppress the vibration and track the target angle. Moreover, comparing with the simulation with PD control, the boundary control laws have ascendancy, i.e., the boundary control laws spend less time on converging to a small scale and have smaller range of elastic deflection than the PD control method. Fig. 13 shows the control input of the boundary control laws. Combining [45] and Fig. 13, we can find that an actual fire-rescue 


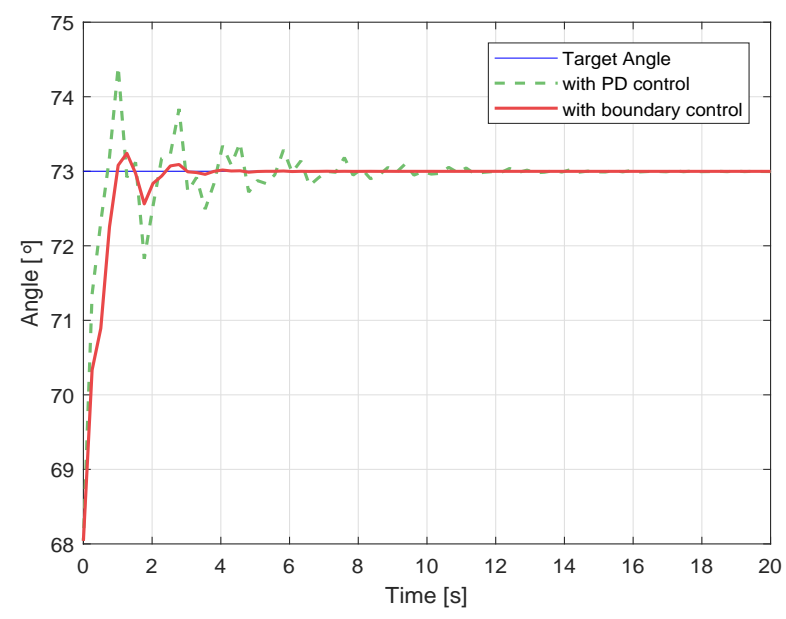

Fig. 11 The angle of the ladder with PD control or boundary control.

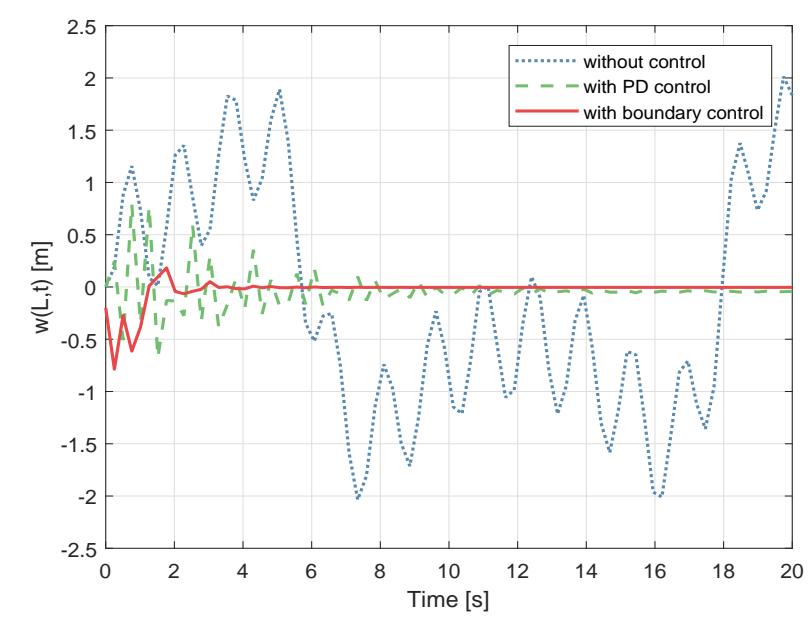

Fig. 12 The vibration offset at the top of the ladder.

ladder can supply enough power to achieve the control objectives.

\section{Conclusion}

This paper uses a segmented Euler-Bernoulli beam with gravity, top mass and external damping to establish an accurate model of a flexible fire-rescue ladder system. Based upon the dynamical model, two boundary controllers are proposed to realise the control goals that the vibration of the closed-loop ladder system is suppressed to a small scale and the angle is able to track the target. By designing a Lyapunov function, the closed-loop ladder system is uniformly ultimately bounded and the elastic deflection $w(z, t)$ remains in a small compact set. Comparing with PD control method, the boundary control strategy can converge to a smaller scale of elastic
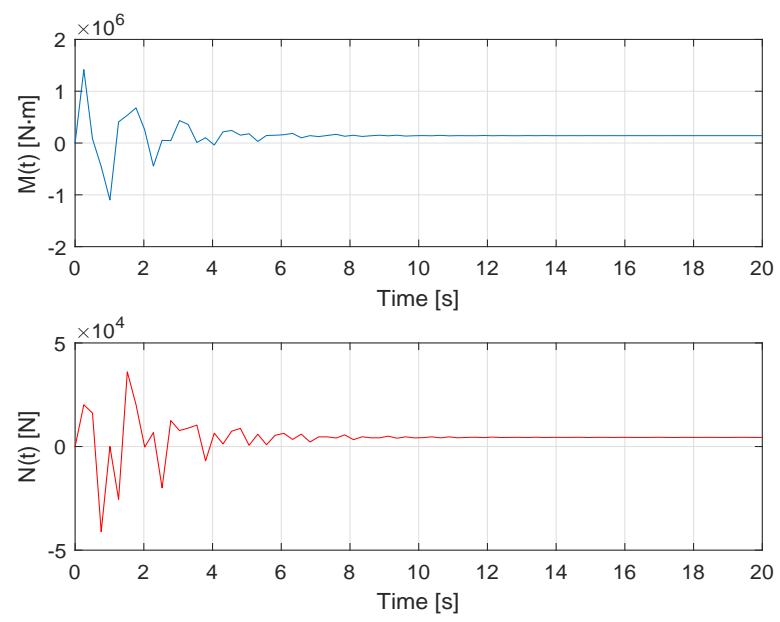

Fig. 13 The control input of the boundary controllers $M(t)$ and $N(t)$.

deflection and track the target angle in less time. Future avenues of research include using adaptive control [30], [44], [19], optimize control [39], neural network control [13], [35], [10], learning-based control methods [31], [14] for flexible fire-rescue ladders, and validating the simulation results to provide more accurate simulations [5], [3], and further giving experiments [18].

\section{Compliance with ethical standards}

Conflict of interest The authors declare that we have no conflict of interest.

Acknowledgements The authors would like to thank the useful comments and constructive suggestions from the handing editor and anonymous reviewers. This work was supported in part by the National Key Research and Development Program of China under Grant 2019YFB1703600, the National Natural Science Foundation of China under Grant 61873297, Joint Funds of Equipment Pre-Research and Ministry of Education of China under Grant 6141A02033339, the Fundamental Research Funds for the China Central Universities under Grant FRF-TP-19-001B2, the China Postdoctoral Science Foundation under Grant 2019TQ0029, the Scientific and Technological Innovation Foundation of Shunde Graduate School of University of Science and Technology Beijing under Grant BK19BE015 and this work was supported by Beijing Top Discipline for Artificial Intelligent Science and Engineering, University of Science and Technology Beijing.

\section{References}

1. Andrianov, I.V., Danishevs' kyy, V.V., Topol, H., Weichert, D.: Homogenization of a 1D nonlinear dynamical problem for periodic composites. ZAMM-Journal of Applied Mathematics and Mechanics/Zeitschrift für Angewandte Mathematik und Mechanik 91(6), 523-534 (2011) 
2. Awrejcewicz, J., Krysko, A., Pavlov, S., Zhigalov, M., Krysko, V.: Chaotic dynamics of size dependent Timoshenko beams with functionally graded properties along their thickness. Mechanical Systems \& Signal Processing 93, 415-430

3. Awrejcewicz, J., Krysko, A.V., Mrozowski, J., Saltykova, O.A., Zhigalov, M.V.: Analysis of regular and chaotic dynamics of the Euler-Bernoulli beams using finite difference and finite element methods. Acta Mechanica Sinica $\mathbf{2 7}(1), 36-43$

4. Awrejcewicz, J., Krysko, A.V., Soldatov, V., Krysko, V.A.: Analysis of the nonlinear dynamics of the Timoshenko flexible beams using wavelets. Journal of Computational \& Nonlinear Dynamics 7(1), 011005

5. Awrejcewicz, J., Krysko, A.V., Zagniboroda, N.A., Dobriyan, V.V., Krysko, V.A.: On the general theory of chaotic dynamics of flexible curvilinear Euler-Bernoulli beams. Nonlinear Dynamics 79(1), 11-29

6. Awrejcewicz, J., Saltykova, O.A., Zhigalov, M.V., Hagedorn, P.: Analysis of non-linear vibrations of singlelayered Euler-Bernoulli beams using wavelets. International Journal of Aerospace and Lightweight Structures (IJALS) 1(2), 203-219

7. Do, K.D.: Stochastic boundary control design for extensible marine risers in three dimensional space. Automatica 77, 184-197 (2017). DOI 10.1016/j.automatica.2016.11.032

8. Endo, T., Matsuno, F., Jia, Y.: Boundary cooperative control by flexible Timoshenko arms. Automatica 81, 377-389 (2017). DOI 10.1016/j.automatica.2017.04.017

9. Endo, T., Sasaki, M., Matsuno, F., Jia, Y.: Contact-force control of a flexible timoshenko arm in rigid/soft environment. IEEE Transactions on Automatic Control 62(5), 2546-2553 (2017). DOI 10.1109/TAC.2016.2599434

10. Gao, H., He, W., Zhou, C., Sun, C.: Neural network control of a two-link flexible robotic manipulator using assumed mode method. IEEE Transactions on Industrial Informatics PP(99), 1-1 (2018)

11. Guo, B.Z., Wang, J., Yang, K.: Dynamic stabilization of an Euler-Bernoulli beam under boundary control and non-collocated observation. Systems \& Control Letters 57(9), 740-749 (2008). DOI 10.1016/j.sysconle.2008.02.004

12. Guo, W., Guo, B.Z.: Performance output tracking for a wave equation subject to unmatched general boundary harmonic disturbance. Automatica 68, 194-202 (2016). DOI 10.1016/j.automatica.2016.01.041

13. He, W., Dong, Y.: Adaptive fuzzy neural network control for a constrained robot using impedance learning. IEEE Transactions on Neural Networks \& Learning Systems 29(4), 1174-1186 (2018)

14. He, W., Gao, H., Zhou, C., Yang, C., Li, Z.: Reinforcement learning control of a flexible manipulator: An experimental investigation. IEEE Transactions on Systems, Man, and Cybernetics: Systems (in press, 2020). DOI 10.1109/TSMC.2020.2975232

15. He, W., Meng, T., He, X., Sun, C.: Iterative learning control for a flapping wing micro aerial vehicle under distributed disturbances. IEEE Transactions on Cybernetics 49(4), 1524-1535 (2019)

16. He, W., Meng, T., Huang, D., Li, X.: Adaptive boundary iterative learning control for an Euler-Bernoulli beam system with input constraint. IEEE Transactions on Neural Networks \& Learning Systems 29(5), 1539-1549 (2018)

17. He, W., Ouyang, Y., Hong, J.: Vibration control of a flexible robotic manipulator in the presence of input deadzone. IEEE Transactions on Industrial Informatics 13(1), 48-59 (2017)
18. He, W., Xue, C., Yu, X., Li, Z., Yang, C.: Admittancebased controller design for physical human-robot interaction in the constrained task space. IEEE Transactions on Automation Science and Engineering (2020). DOI 10.1109/TASE.2020.2983225

19. He, X., He, W., Liu, Y., Wang, Y., Li, G., Wang, Y.: Robust adaptive control of an offshore ocean thermal energy conversion system. IEEE Transactions on Systems Man \& Cybernetics Systems (2018). DOI 10.1109/TSMC.2018.2870999

20. He, X., He, W., Shi, J., Sun, C.: Boundary vibration control of variable length crane systems in two dimensional space with output constraints. IEEE/ASME Transactions on Mechatronics 22(5), 1952-1962 (2017). DOI 10.1109/TMECH.2017.2721553

21. Huang, D., Xu, J.X., Li, X., Xu, C., Yu, M.: D-type anticipatory iterative learning control for a class of inhomogeneous heat equations. Automatica 49(8), 2397-2408 (2013). DOI 10.1016/j.automatica.2013.05.005

22. Ji, N., Liu, Z., Liu, J., He, W.: Vibration control for a nonlinear three-dimensional Euler-Bernoulli beam under input magnitude and rate constraints. Nonlinear Dynamics 91(4), 2551-2570 (2018). DOI 10.1007/s11071017-4031-y

23. Krstic, M., Guo, B.Z., Balogh, A., Smyshlyaev, A.: Output-feedback stabilization of an unstable wave equation. Automatica 44(1), 63-74 (2008). DOI 10.1016/j.automatica.2007.05.012

24. Krstic, M., Smyshlyaev, A.: Boundary control of PDEs: A course on backstepping designs, vol. 16. Siam (2008)

25. Lambeck, S., Sawodny, O., Arnold, E.: Trajectory tracking control for a new generation of fire rescue turntable ladders. In: 2006 IEEE Conference on Robotics, Automation and Mechatronics, pp. 1-6 (Bangkok, Thailand, 2006)

26. Liu, Y., Fu, Y., He, W., Hui, Q.: Modeling and observerbased vibration control of a flexible spacecraft with external disturbances. IEEE Transactions on Industrial Electronics 66(11), 8648-8658 (2019)

27. Liu, Z., Liu, J., He, W.: Modeling and vibration control of a flexible aerial refueling hose with variable lengths and input constraint. Automatica 77, 302-310 (2017)

28. Liu, Z., Zhao, Z., Ahn, C.K.: Boundary constrained control of flexible string systems subject to disturbances. IEEE Transactions on Circuits and Systems II: Express Briefs 67(1), 112-116 (2020)

29. Luo, Z.H., Guo, B.Z., Morgül, O.: Stability and Stabilization of Infinite Dimensional Systems with Applications. Springer London (1999)

30. Mu, C., Ni, Z., Sun, C., He, H.: Air-breathing hypersonic vehicle tracking control based on adaptive dynamic programming. IEEE Transactions on Neural Networks and Learning Systems 28(3), 584-598 (2016)

31. $\mathrm{Mu}, \mathrm{C}$. , Zhang, Y.: Learning-based robust tracking control of quadrotor with time-varying and coupling uncertainties. IEEE Transactions on Neural Networks and Learning Systems 31(1), 259-273 (2020)

32. Pertsch, A., Zimmert, N., Sawodny, O.: Modeling a fire-rescue turntable ladder as piecewise Euler-Bernoulli beam with a tip mass. In: Proceedings of the 48th IEEE Conference on Decision and Control, 2009 Held Jointly with the 2009 28th Chinese Control Conference (CDC/CCC 2009), pp. 7321-7326 (Shanghai, China, 2009). DOI 10.1109/CDC.2009.5399815

33. Rahn, C.D.: Mechatronic control of distributed noise and vibration. Automatica 39(9), 1664-1666 (2003) 
34. Sawodny, O., Lambeck, S., Hildebrandt, A.: Trajectory generation for the trajectory tracking control of a fire rescue turntable ladder. In: International Workshop on Robot Motion and Control, pp. 411-416 (Poland, 2002). DOI 10.1109/ROMOCO.2002.1177141

35. Sun, C., He, W., Hong, J.: Neural network control of a flexible robotic manipulator using the lumped springmass model. IEEE Transactions on Systems Man \& Cybernetics Systems 47(8), 1863-1874 (2017)

36. Sun, N., Fang, Y.: Nonlinear tracking control of underactuated cranes with load transferring and lowering: Theory and experimentation . Automatica 50(9), 2350-2357 (2014)

37. Wang, J.M., Ren, B., Krstic, M.: Stabilization and Gevrey regularity of a Schrödinger equation in boundary feedback with a heat equation. IEEE Transactions on Automatic Control 57(1), 179-185 (2012). DOI 10.1109/TAC.2011.2164299

38. Wu, H.N., Wang, J.W.: Static output feedback control via PDE boundary and ODE measurements in linear cascaded ODE-beam systems. Automatica 50(11), 2787-2798 (2014). DOI 10.1016/j.automatica.2014.09.006

39. Wu, X., Xu, L., Wang, J., Yang, D., Li, F., Li, X.: A prognostic-based dynamic optimization strategy for a degraded solid oxide fuel cell. Sustainable Energy Technologies and Assessments 39, 100682 (2020)

40. Yang, K.J., Hong, K.S., Matsuno, F.: Robust adaptive boundary control of an axially moving string under a spatiotemporally varying tension. Journal of Sound \& Vibration 273(4), 1007-1029 (2004). DOI 10.1016/S0022460X(03)00519-4

41. Yang, K.J., Hong, K.S., Matsuno, F.: Robust boundary control of an axially moving string by using a pr transfer function. IEEE Transactions on Automatic Control 50(12), 2053-2058 (2005)

42. Zhao, Z., Ahn, C.K., Li, H.X.: Boundary anti-disturbance control of a spatially nonlinear flexible string system. IEEE Transactions on Industrial Electronics 67(6), 48464856 (2020)

43. Zhao, Z., Lin, S., Zhu, D., Wen, G.: Vibration control of a riser-vessel system subject to input backlash and extraneous disturbances. IEEE Transactions on Circuits and Systems II: Express Briefs 67(3), 516-520 (2020)

44. Zhou, Q., Li, H., Wang, L., Lu, R.: Prescribed performance observer-based adaptive fuzzy control for nonstrict-feedback stochastic nonlinear systems. IEEE Transactions on Systems, Man, and Cybernetics: Systems 48(10), 1747-1758 (2017)

45. Zimmert, N., Pertsch, A., Sawodny, O.: 2-DOF control of a fire-rescue turntable ladder. IEEE Transactions on Control Systems Technology 20(2), 438-452 (2012). DOI 10.1109/TCST.2011.2116021

\section{Appendices}

\section{A. Proof of Step 1}

Proof : Using the Young's inequality and (10)-(12) to $V_{I I I}(t)$, we have

$$
\left|V_{I I I}(t)\right| \leq \alpha \sum_{n=1}^{S} \int_{z_{n-1}^{+}}^{z_{n}^{-}} \rho_{n}|w(z, t)||\dot{P}(z, t)| d z
$$

$$
\begin{aligned}
& +\xi \sum_{n=1}^{S} \int_{z_{n-1}^{+}}^{z_{n}^{-}} \rho_{n} z\left|w^{\prime}(z, t)\right||\dot{P}(z, t)| d z \\
& +\zeta \sum_{n=1}^{S} \int_{z_{n-1}^{+}}^{z_{n}^{-}} \rho_{n}(L-z)\left|w^{\prime}(z, t)\right||\dot{P}(z, t)| d z \\
\leq & \sum_{n=1}^{S} \int_{z_{n-1}^{+}}^{z_{n}^{-}} L \rho_{\max }\left[2 L \alpha \delta_{1}+(\zeta+\xi) \delta_{2}\right] \\
& \times\left[w^{\prime \prime}(z, t)\right]^{2} d z+\sum_{n=1}^{S} \int_{z_{n-1}^{+}}^{z_{n}^{-}}\left[\frac{(\zeta+\xi) \rho_{n}}{2 \delta_{2}}\right. \\
& \left.+\frac{\alpha \rho_{n}}{2 \delta_{1}}\right][\dot{P}(z, t)]^{2} d z \\
\leq & \mu_{1} V_{1}(t)
\end{aligned}
$$

where $\delta_{1}$ and $\delta_{2}$ are positive constants, $\rho_{\max }$ is the maximum value of $\rho_{n}, n \in\{1,2, \ldots, S\}$ and

$$
\begin{gathered}
\mu_{1}=\max \left\{\frac{2 L \rho_{\max }\left[2 L \alpha \delta_{1}+(\zeta+\xi) \delta_{2}\right]}{\beta E I_{\min }},\right. \\
\left.\frac{(\zeta+\xi)}{\beta \delta_{2}}+\frac{\alpha}{\beta \delta_{1}}\right\}
\end{gathered}
$$

where $E I_{\min }$ is the minimum value of $E I_{n}, n \in\{1,2$, $\ldots, S\}$. Therefore, we obtain

$-\mu_{1} V_{1}(t) \leq V_{3}(t) \leq \mu_{1} V_{1}(t)$

When $\mu_{1}$ satisfies $0<\mu_{1}<1$, we have

$0 \leq \lambda_{1}\left(V_{1}(t)+V_{2}(t)\right) \leq V(t) \leq \lambda_{2}\left(V_{I}(t)+V_{I I}(t)\right)$

where $\lambda_{1}=1-\mu_{1}$ and $\lambda_{2}=1+\mu_{1}$.

\section{B. Proof of Step 2}

Proof : Taking the derivative of (15) with respect to time, we have

$\dot{V}(t)=\dot{V}_{I}(t)+\dot{V}_{I I}(t)+\dot{V}_{I I I}(t)$.

Using the motion equation (2), the first term of (B.1) can be written as

$$
\begin{aligned}
\dot{V}_{I}(t)= & -\beta \sum_{n=1}^{S} \int_{z_{n-1}^{+}}^{z_{n}^{-}} \rho_{n} g \dot{P}(z, t) \cos \theta(t) d z \\
& -\beta E I_{S} \dot{P}(L, t) P^{\prime \prime \prime}(L, t)-\beta E I_{1} P^{\prime \prime}(0, t) \dot{\theta}(t) \\
& -\beta \sum_{n=1}^{S} \int_{z_{n-1}^{+}}^{z_{n}^{-}} c[\dot{P}(z, t)]^{2} d z .
\end{aligned}
$$

We define

$$
\begin{aligned}
& B_{1}(t)=-\beta \sum_{n=1}^{S} \int_{z_{n-1}^{+}}^{z_{n}^{-}} \rho_{n} g \dot{P}(z, t) \cos \theta(t) d z, \\
& B_{2}(t)=-\beta E I_{1} P^{\prime \prime}(0, t) \dot{\theta}(t) .
\end{aligned}
$$


Using the Young's inequality, we have

$$
\begin{aligned}
\left|B_{1}\right| \leq & \beta \sum_{n=1}^{S} \int_{z_{n-1}^{+}}^{z_{n}^{-}} \rho_{n} g \frac{\delta_{3}}{2}[\dot{P}(z, t)]^{2} d z \\
& +\beta \sum_{n=1}^{S} \int_{z_{n-1}^{+}}^{z_{n}^{-}} \rho_{n} g \frac{1}{2 \delta_{3}}[\cos \theta(t)]^{2} d z \\
\leq & \beta \sum_{n=1}^{S} \int_{z_{n-1}^{+}}^{z_{n}^{-}} \rho_{n} g \frac{\delta_{3}}{2}[\dot{P}(z, t)]^{2} d z+\frac{\beta g L \rho_{\max }}{2 \delta_{3}} \\
\left|B_{2}\right| \leq & \beta E I_{1} \frac{\delta_{4}}{2}\left[P^{\prime \prime}(0, t)\right]^{2}+\beta E I_{1} \frac{1}{2 \delta_{4}}[\dot{\theta}(t)]^{2},
\end{aligned}
$$

where $\delta_{3}$ and $\delta_{4}$ are positive constants. As a result, we obtain

$$
\begin{aligned}
\dot{V}_{I}(t) \leq & \beta \sum_{n=1}^{S} \int_{z_{n-1}^{+}}^{z_{n}^{-}} \rho_{n} g \frac{\delta_{3}}{2}[\dot{P}(z, t)]^{2} d z+\beta E I_{1} \frac{1}{2 \delta_{4}}[\dot{\theta}]^{2} \\
& +\beta E I_{1} \frac{\delta_{4}}{2}\left[P^{\prime \prime}(0, t)\right]^{2}-\beta \sum_{n=1}^{S} \int_{z_{n-1}^{+}}^{z_{n}^{-}} c[\dot{P}(z, t)]^{2} d z \\
& -\beta E I_{S} \dot{P}(L, t) P^{\prime \prime \prime}(L, t)+\frac{\beta g L \rho_{\max }}{2 \delta_{3}} .
\end{aligned}
$$

Using the motion equation (3), the second term of (B.1) can be written as

$$
\begin{aligned}
\dot{V}_{I I}(t)= & \gamma \sum_{n=1}^{S} \int_{z_{n-1}^{+}}^{z_{n}^{-}} \rho_{n} g w(z, t)\left[\dot{\theta}(t)+\theta_{e}(t)\right] \sin \theta(t) d z \\
& +\gamma\left[\dot{\theta}(t)+\theta_{e}(t)\right]\left[M(t)+E I_{1} P^{\prime \prime}(0, t)\right. \\
& \left.+m_{c} g w(L, t) \sin \theta(t)+J_{A} \dot{\theta}(t)\right] \\
& +\beta \alpha^{2} m_{c} u_{a}(t) \dot{u}_{a}(t)+k \theta_{e}(t) \dot{\theta}(t) .
\end{aligned}
$$

Using perfect square formula, we have

$$
k \theta_{e}(t) \dot{\theta}(t)=\frac{k}{2}\left[\theta_{e}(t)+\dot{\theta}(t)\right]^{2}-\frac{k}{2}\left[\theta_{e}(t)\right]^{2}-\frac{k}{2}[\dot{\theta}(t)]^{2}
$$

Using the definition of $u_{a}(t)$ and the boundary condition (9), we have

$$
\begin{aligned}
\beta \alpha m_{c} u_{a}(t) \dot{u}_{a}(t)= & \beta \alpha m_{c} u_{a}(t)\left[\ddot{P}(L, t)+\frac{\alpha-\xi L}{\beta} \dot{w}(L, t)\right] \\
= & \beta \alpha u_{a}(t)\left[N(t)+E I_{S} P^{\prime \prime \prime}(L, t)\right. \\
& \left.\left.-m_{c} g \cos \theta(t)+m_{c} \frac{\alpha-\xi L}{\beta} \dot{w}(I(B) .)\right] 0\right)
\end{aligned}
$$

Defining $B_{3}=\gamma \sum_{n=1}^{S} \int_{z_{n-1}^{+}}^{z_{n}^{-}} \rho_{n} g w(z, t)\left[\dot{\theta}(t)+\theta_{e}(t)\right]$ $\times \sin \theta(t) d z$ and using the Young's inequality and (10)-
(12), we have

$$
\begin{aligned}
\left|B_{3}\right| \leq & \gamma \sum_{n=1}^{S} \int_{z_{n-1}^{+}}^{z_{n}^{-}} \rho_{n} g|w(z, t)|\left|\left[\dot{\theta}(t)+\theta_{e}(t)\right]\right| d z \\
\leq & \gamma \sum_{n=1}^{S} \int_{z_{n-1}^{+}}^{z_{n}^{-}} \rho_{n} g \frac{\delta_{5}}{2}[w(z, t)]^{2} d z \\
& +\gamma \sum_{n=1}^{S} \int_{z_{n-1}^{+}}^{z_{n}^{-}} \rho_{n} g \frac{1}{2 \delta_{5}}\left[\dot{\theta}(t)+\theta_{e}(t)\right]^{2} d z \\
\leq & \gamma \sum_{n=1}^{S} \int_{z_{n-1}^{+}}^{z_{n}^{-}} 2 L^{2} \rho_{n} g \delta_{5}\left[w^{\prime \prime}(z, t)\right]^{2} d z \\
& +\frac{\gamma g \rho_{\max } L}{2 \delta_{5}}\left[\dot{\theta}(t)+\theta_{e}(t)\right]^{2},
\end{aligned}
$$

where $\delta_{5}$ is positive constant. Substituting (B.9)-(B.11) and the boundary control laws (13) and (14) to (B.8), we further have

$$
\begin{aligned}
\dot{V}_{I I}(t) \leq & \gamma \sum_{n=1}^{S} \int_{z_{n-1}^{+}}^{z_{n}^{-}} 2 L^{2} \rho_{n} g \delta_{5}\left[w^{\prime \prime}(z, t)\right]^{2} d z-\frac{k}{2}\left[\theta_{e}(t)\right]^{2} \\
& -\frac{k}{2}[\dot{\theta}(t)]^{2}-k_{u}\left[u_{a}(t)\right]^{2}+\sqrt{\beta} u_{a}(t) E I_{S} P^{\prime \prime \prime}(L, t) \\
& -\left(k_{\theta}-\frac{\gamma g \rho_{\max } L}{2 \delta_{5}}\right)\left[\dot{\theta}(t)+\theta_{e}(t)\right]^{2} .
\end{aligned}
$$

The third term of (B.1) can be written as

$\dot{V}_{I I I}(t)=B_{4}+B_{5}+B_{6}+B_{7}+B_{8}+B_{9}$,

where

$$
\begin{aligned}
& B_{4}=\alpha \sum_{n=1}^{S} \int_{z_{n-1}^{+}}^{z_{n}^{-}} \rho_{n} \dot{w}(z, t) \dot{P}(z, t) d z \\
& B_{5}=\alpha \sum_{n=1}^{S} \int_{z_{n-1}^{+}}^{z_{n}^{-}} \rho_{n} w(z, t) \ddot{P}(z, t) d z
\end{aligned}
$$$$
B_{6}=\zeta \sum_{n=1}^{S} \int_{z_{n-1}^{+}}^{z_{n}^{-}} \rho_{n}(L-z) \dot{w}^{\prime}(z, t) \dot{P}(z, t) d z
$$$$
B_{7}=\zeta \sum_{n=1}^{S} \int_{z_{n-1}^{+}}^{z_{n}^{-}} \rho_{n}(L-z) w^{\prime}(z, t) \ddot{P}(z, t) d z
$$

$B_{8}=-\xi \sum_{n=1}^{S} \int_{z_{n-1}^{+}}^{z_{n}^{-}} \rho_{n} z \dot{w}^{\prime}(z, t) \dot{P}(z, t) d z$

$B_{9}=-\xi \sum_{n=1}^{S} \int_{z_{n-1}^{+}}^{z_{n}^{-}} \rho_{n} z w^{\prime}(z, t) \ddot{P}(z, t) d z$

Using the definition of $P(z, t), B_{4}$ can be written as $B_{4}=\alpha \sum_{n=1}^{S} \int_{z_{n-1}^{+}}^{z_{n}^{-}} \rho_{n}[\dot{P}(z, t)]^{2} d z$

$$
-\alpha \sum_{n=1}^{S} \int_{z_{n-1}^{+}}^{z_{n}^{-}} \rho_{n} z \dot{P}(z, t) \dot{\theta}(t) d z \text {. }
$$


Using the Young's inequality, the seconde term of $B_{4}$ satisfies following inequality.

$$
\begin{aligned}
& \left|\alpha \sum_{n=1}^{S} \int_{z_{n-1}^{+}}^{z_{n}^{-}} \rho_{n} z \dot{P}(z, t) \dot{\theta}(t) d z\right| \\
\leq & \sum_{n=1}^{S} \int_{z_{n-1}^{+}}^{z_{n}^{-}} \frac{\delta_{6} \alpha \rho_{n}}{2} z[\dot{P}(z, t)]^{2} d z \\
& +\frac{\alpha}{2 \delta_{6}} \sum_{n=1}^{S} \int_{z_{n-1}^{+}}^{z_{n}^{-}} \rho_{n} z[\dot{\theta}(t)]^{2} d z \\
\leq & \sum_{n=1}^{S} \int_{z_{n-1}^{+}}^{z_{n}^{-}} \frac{\delta_{6} \alpha \rho_{n} L}{2}[\dot{P}(z, t)]^{2} d z \frac{\alpha \rho_{\max } L^{2}}{4 \delta_{6}}[\dot{\theta}(t)]^{2},
\end{aligned}
$$

where $\delta_{6}$ is a positive constant. So that $B_{4}$ satisfies

$$
\begin{aligned}
B_{4} \leq & \alpha \sum_{n=1}^{S} \int_{z_{n-1}^{+}}^{z_{n}^{-}} \rho_{n}\left(1+\frac{\delta_{6} L}{2}\right)[\dot{P}(z, t)]^{2} d z \\
& +\frac{\alpha \rho_{\max } L^{2}}{4 \delta_{6}}[\dot{\theta}(t)]^{2} .
\end{aligned}
$$

Using the motion equation (2), $B_{5}$ can be written as

$$
\begin{aligned}
B_{5}= & -\alpha \sum_{n=1}^{S} \int_{z_{n-1}^{+}}^{z_{n}^{-}} \rho_{n} g w(z, t) \cos \theta(t) d z-\alpha E I_{S} w(L, t) \\
& \times P^{\prime \prime \prime}(L, t)-\alpha \sum_{n=1}^{S} \int_{z_{n-1}^{+}}^{z_{n}^{-}} E I_{n}\left[w^{\prime \prime}(z, t)\right]^{2} d z \\
& -\alpha c \sum_{n=1}^{S} \int_{z_{n-1}^{+}}^{z_{n}^{-}} w(z, t) \dot{P}(z, t) d z
\end{aligned}
$$

Using the Young's inequality, (10) and (11), the first term of $B_{5}$ satisfies following inequality.

$$
\begin{aligned}
& \left|\alpha \sum_{n=1}^{S} \int_{z_{n-1}^{+}}^{z_{n}^{-}} \rho_{n} g w(z, t) \cos \theta(t) d z\right| \\
\leq & \alpha \sum_{n=1}^{S} \int_{z_{n-1}^{+}}^{z_{n}^{-}} \rho_{n} g \frac{\delta_{7}}{2}[w(z, t)]^{2} d z+\frac{\alpha}{2 \delta_{7}} \sum_{n=1}^{S} \int_{z_{n-1}^{+}}^{z_{n}^{-}} \rho_{n} g d z \\
\leq & 2 L^{2} \delta_{7} g \alpha \sum_{n=1}^{S} \int_{z_{n-1}^{+}}^{z_{n}^{-}} \rho_{\max }\left[w^{\prime \prime}(z, t)\right]^{2} d z \\
& +\frac{\alpha \rho_{\max } L g}{2 \delta_{7}}
\end{aligned}
$$

where $\delta_{7}$ is a positive constant. Using the Young's inequality and (10), the forth term of $B_{5}$ satisfies following inequality.

$$
\begin{aligned}
& \left|\alpha c \sum_{n=1}^{S} \int_{z_{n-1}^{+}}^{z_{n}^{-}} w(z, t) \dot{P}(z, t) d z\right| \\
\leq & \alpha \sum_{n=1}^{S} \int_{z_{n-1}^{+}}^{z_{n}^{-}} 2 c L^{2} \delta_{8}\left[w^{\prime \prime}(z, t)\right]^{2} d z \\
& +\frac{c \alpha}{2 \delta_{8}} \sum_{n=1}^{S} \int_{z_{n-1}^{+}}^{z_{n}^{-}}[\dot{P}(z, t)]^{2} d z,
\end{aligned}
$$

where $\delta_{8}$ is a positive constant. So that $B_{5}$ satisfies

$$
\begin{aligned}
B_{5} \leq & -\alpha E I_{S} w(L, t) P^{\prime \prime \prime}(L, t)+\frac{\alpha \rho_{\max } L g}{2 \delta_{7}} \\
& +\frac{c \alpha}{2 \delta_{8}} \sum_{n=1}^{S} \int_{z_{n-1}^{+}}^{z_{n}^{-}}[\dot{P}(z, t)]^{2} d z-\alpha \sum_{n=1}^{S} \int_{z_{n-1}^{+}}^{z_{n}^{-}}\left(E I_{n}\right. \\
& \left.-2 L^{2} \delta_{7} g \rho_{\max }-2 L^{2} c \delta_{8}\right)\left[w^{\prime \prime}(z, t)\right]^{2} d z
\end{aligned}
$$

Using the definition of $P(z, t)$ and integrating by part, $B_{6}$ can be written as

$$
\begin{aligned}
B_{6}= & \zeta \sum_{n=1}^{S} \int_{z_{n-1}^{+}}^{z_{n}^{-}} \rho_{n}(L-z) \dot{P}^{\prime}(z, t) \dot{P}(z, t) d z \\
& -\zeta \sum_{n=1}^{S} \int_{z_{n-1}^{+}}^{z_{n}^{-}} \rho_{n}(L-z) \dot{\theta}(t) \dot{P}(t) d z \\
= & \zeta \sum_{n=1}^{S-1}\left(\rho_{n}-\rho_{n+1}\right)\left(L-z_{n}^{-}\right)\left[\dot{P}\left(z_{n}^{-}, t\right)\right]^{2} \\
& -\zeta \sum_{n=1}^{S} \int_{z_{n-1}^{+}}^{z_{n}^{-}} \rho_{n}(L-z) \dot{\theta}(t) \dot{P}(z, t) d z \\
& +\frac{\zeta}{2} \sum_{n=1}^{S} \int_{z_{n-1}^{+}}^{z_{n}^{-}} \rho_{n}[\dot{P}(z, t)]^{2} d z
\end{aligned}
$$

Using the Young's inequality, the term $\zeta \sum_{n=1}^{S} \int_{z_{n-1}^{+}}^{z_{n}^{-}} \rho_{n}$ $\times(L-z) \dot{\theta}(t) \dot{P}(z, t) d z$ in $B_{6}$ satisfies following inequality:

$$
\begin{aligned}
& \left|\zeta \sum_{n=1}^{S} \int_{z_{n-1}^{+}}^{z_{n}^{-}} \rho_{n}(L-z) \dot{\theta}(t) \dot{P}(z, t) d z\right| \\
\leq & \frac{L \zeta \delta_{9}}{2} \sum_{n=1}^{S} \int_{z_{n-1}^{+}}^{z_{n}^{-}} \rho_{n}[\dot{P}(z, t)]^{2} d z+\frac{\zeta \rho_{\max } L^{2}}{2 \delta_{9}}[\dot{\theta}(t)]^{2} d z
\end{aligned}
$$

where $\delta_{9}$ is a positive constant. So that $B_{6}$ satisfies

$$
\begin{aligned}
B_{6} \leq & \zeta \sum_{n=1}^{S-1}\left(\rho_{n}-\rho_{n+1}\right)\left(L-z_{n}^{-}\right)\left[\dot{P}\left(z_{n}^{-}, t\right)\right]^{2}+\frac{\zeta \rho_{\max } L^{2}}{2 \delta_{9}} \\
& \times[\dot{\theta}(t)]^{2} d z+\frac{\zeta+L \zeta \delta_{9}}{2} \sum_{n=1}^{S} \int_{z_{n-1}^{+}}^{z_{n}^{-}} \rho_{n}[\dot{P}(z, t)]^{2} d z
\end{aligned}
$$

Using the motion equation (2), $B_{7}$ can be written as

$$
\begin{aligned}
B_{7}= & -\frac{\zeta}{2} L E I_{1}\left[w^{\prime \prime}(0, t)\right]^{2}-\zeta \sum_{n=1}^{S-1}\left(\rho_{n}-\rho_{n+1}\right) g(L \\
& \left.-z_{n}^{-}\right) w\left(z_{n}^{-}, t\right) \cos \theta(t)-\zeta \sum_{n=1}^{S} \int_{z_{n-1}^{+}}^{z_{n}^{-}} w(z, t) \rho_{n} g \\
& \times \cos \theta(t) d z+\frac{3 \zeta}{2} \sum_{n=1}^{S} \int_{z_{n-1}^{+}}^{z_{n}^{-}} E I_{n}\left[w^{\prime \prime}(z, t)\right]^{2} \\
& -\zeta \sum_{n=1}^{S} \int_{z_{n-1}^{+}}^{z_{n}^{-}}(L-z) w^{\prime}(z, t) c \dot{P}(z, t) d z
\end{aligned}
$$


Considering $\rho_{n}>\rho_{n+1}$ and using the Young's inequality and (12), we have the first term of $B_{7}$ satisfies

$$
\begin{aligned}
& \left|\zeta \sum_{n=1}^{S-1}\left(\rho_{n}-\rho_{n+1}\right) g\left(L-z_{n}^{-}\right) w\left(z_{n}^{-}, t\right) \cos \theta(t)\right| \\
\leq & \zeta \rho_{\max } g L \sum_{n=1}^{S-1}\left|w\left(z_{n}^{-}, t\right) \cos \theta(t)\right| \\
\leq & \frac{\delta_{10} 8 g L^{2}(S-1) \zeta \rho_{\max }}{2} \sum_{n=1}^{S} \int_{z_{n-1}^{+}}^{z_{n}^{-}}\left[w^{\prime \prime}(z, t)\right]^{2} d z \\
& +\frac{g L(S-1) \zeta \rho_{\max }}{2 \delta_{10}}
\end{aligned}
$$

where $\delta_{10}$ is a positive constant. Similar with (B.18), the second term in $B_{7}$ satisfies

$$
\begin{aligned}
& \left|\zeta \sum_{n=1}^{S} \int_{z_{n-1}^{+}}^{z_{n}^{-}} w(z, t) \rho_{n} g \cos \theta(t) d z\right| \\
\leq & 2 L^{2} \delta_{7} g \varsigma \sum_{n=1}^{S} \int_{z_{n-1}^{+}}^{z_{n}^{-}} \rho_{\max }\left[w^{\prime \prime}(z, t)\right]^{2} d z+\frac{\zeta \rho_{\max } L g}{2 \delta_{7}}
\end{aligned}
$$

Using the Young's inequality and (11), the third term of $B_{7}$ satisfies following inequality.

$$
\begin{aligned}
& \left|\zeta \sum_{n=1}^{S} \int_{z_{n-1}^{+}}^{z_{n}^{-}}(L-z) w^{\prime}(z, t) c \dot{P}(z, t) d z\right| \\
\leq & c L^{2} \delta_{11} \zeta \sum_{n=1}^{S} \int_{z_{n-1}^{+}}^{z_{n}^{-}}\left[w^{\prime \prime}(z, t)\right]^{2} d z \\
& +\frac{c L \zeta}{2 \delta_{11}} \sum_{n=1}^{S} \int_{z_{n-1}^{+}}^{z_{n}^{-}}[\dot{P}(z, t)]^{2} d z
\end{aligned}
$$

where $\delta_{12}$ is a positive constant. So that $B_{7}$ satisfies

$$
\begin{aligned}
B_{7} \leq & \zeta \sum_{n=1}^{S} \int_{z_{n-1}^{+}}^{z_{n}^{-}}\left(2 L^{2} \delta_{7} g \rho_{\max }+\frac{8(S-1) \rho_{\max } \delta_{10} g L^{2}}{2}\right. \\
& \left.+\frac{3}{2} E I_{n}+c L^{2} \delta_{11}\right)\left[w^{\prime \prime}(z, t)\right]^{2} d z+\frac{\zeta \rho_{\max } L g}{2 \delta_{7}} \\
& +\frac{(S-1) \zeta \rho_{\max } g L}{2 \delta_{10}}+\frac{c L \zeta}{2 \delta_{11}} \sum_{n=1}^{S} \int_{z_{n-1}^{+}}^{z_{n}^{-}}[\dot{P}(z, t)]^{2} d z \\
& -\frac{\zeta}{2} L E I_{1}\left[w^{\prime \prime}(0, t)\right]^{2}
\end{aligned}
$$

Using the definition of $P(z, t)$ and integrating by part, $B_{8}$ can be written as

$$
\begin{aligned}
B_{8}= & -\xi \sum_{n=1}^{S} \int_{z_{n-1}^{+}}^{z_{n}^{-}} \rho_{n} z \dot{P}^{\prime}(z, t) \dot{P}(z, t) d z \\
& +\xi \sum_{n=1}^{S} \int_{z_{n-1}^{+}}^{z_{n}^{-}} \rho_{n} z \dot{\theta}(t) \dot{P}(t) d z
\end{aligned}
$$

$$
\begin{aligned}
= & -\frac{\xi}{2} \sum_{n=1}^{S-1}\left(\rho_{n}-\rho_{n+1}\right) z_{n}^{-}\left[\dot{P}\left(z_{n}^{-}, t\right)\right]^{2} \\
& -\frac{\xi}{2} \sum_{n=1}^{S} \rho_{S} L[\dot{P}(L, t)]^{2}+\frac{\xi}{2} \sum_{n=1}^{S} \int_{z_{n-1}^{+}}^{z_{n}^{-}} \rho_{n}[\dot{P}(z, t)]^{2} d z \\
& +\xi \sum_{n=1}^{S} \int_{z_{n-1}^{+}}^{z_{n}^{-}} \rho_{n} z \dot{\theta}(t) \dot{P}(z, t) d z
\end{aligned}
$$

Using the Young's inequality, the forth term of $B_{8}$ satisfies following inequality.

$$
\begin{aligned}
& \left|\xi \sum_{n=1}^{S} \int_{z_{n-1}^{+}}^{z_{n}^{-}} \rho_{n} z \dot{P}(z, t) \dot{\theta}(t) d z\right| \\
\leq & \sum_{n=1}^{S} \int_{z_{n-1}^{+}}^{z_{n}^{-}} \frac{\delta_{12} \xi \rho_{n} L}{2}[\dot{P}(z, t)]^{2} d z+\frac{\xi \rho_{\max } L^{2}}{4 \delta_{12}}[\dot{\theta}(t)]^{2}
\end{aligned}
$$

where $\delta_{13}$ is a positive constant. So that $B_{8}$ satisfies

$$
\begin{aligned}
B_{8} \leq & \frac{\xi}{2} \sum_{n=1}^{S} \int_{z_{n-1}^{+}}^{z_{n}^{-}} \rho_{n}\left(1+\delta_{12} L\right)[\dot{P}(z, t)]^{2} d z-\frac{\xi}{2} \sum_{n=1}^{S} \rho_{S} L \\
& \times[\dot{P}(L, t)]^{2}-\frac{\xi}{2} \sum_{n=1}^{S-1}\left(\rho_{n}-\rho_{n+1}\right) z_{n}^{-}\left[\dot{P}\left(z_{n}^{-}, t\right)\right]^{2} \\
& +\frac{\xi \rho_{\max } L^{2}}{4 \delta_{12}}[\dot{\theta}(t)]^{2}
\end{aligned}
$$

Using the motion equation (2) and integrating by part, $B_{9}$ can be written as

$$
\begin{aligned}
B_{9}= & \xi \sum_{n=1}^{S-1}\left(\rho_{n}-\rho_{n+1}\right) z_{n}^{-} w\left(z_{n}^{-}, t\right) \cos \theta(t)-\sum_{n=1}^{S} \int_{z_{n-1}^{+}}^{z_{n}^{-}} \xi \\
& \times \rho_{n} g w(z, t) \cos \theta(t) d z+\xi \rho_{S} L w(L, t) \cos \theta(t) \\
& +\xi E I_{S} L w(L, t) P^{\prime \prime \prime}(L, t)+\xi \sum_{n=1}^{S} \int_{z_{n-1}^{+}}^{z_{n}^{-}} c z w^{\prime}(z, t) \\
& \times \dot{P}(z, t) d z+\frac{3 \xi}{2} \sum_{n=1}^{S} \int_{z_{n-1}^{+}}^{z_{n}^{-}} E I_{n}\left[w^{\prime \prime}(z, t)\right]^{2} d z(\mathrm{~B} .32)
\end{aligned}
$$

Similar with (B.25), (B.18) and (B.27), the first, third and sixth terms of $B_{9}$ satisfy respectively following inequalities:

$$
\begin{aligned}
& \left|\xi \sum_{n=1}^{S-1}\left(\rho_{n}-\rho_{n+1}\right) z_{n}^{-} w\left(z_{n}^{-}, t\right) \cos \theta(t)\right| \\
\leq & \frac{8(S-1) \delta_{10} \xi \rho_{\max } g L^{2}}{2} \sum_{n=1}^{S} \int_{z_{n-1}^{+}}^{z_{n}^{-}}\left[w^{\prime \prime}(z, t)\right]^{2} d z \\
+ & \frac{(S-1) \xi \rho_{\max } g L}{2 \delta_{10}}
\end{aligned}
$$




$$
\begin{aligned}
& \left|\xi \sum_{n=1}^{S} \int_{z_{n-1}^{+}}^{z_{n}^{-}} \rho_{n} g w(z, t) \cos \theta(t) d z\right| \\
\leq & 2 L^{2} \delta_{7} g \xi \sum_{n=1}^{S} \int_{z_{n-1}^{+}}^{z_{n}^{-}} \rho_{\max }\left[w^{\prime \prime}(z, t)\right]^{2} d z+\frac{\xi \rho_{\max } L g}{2 \delta_{7}} \\
& \left|\xi \sum_{n=1}^{S} \int_{z_{n-1}^{+}}^{z_{n}^{-}} z w^{\prime}(z, t) c \dot{P}(z, t) d z\right| \\
\leq & c L^{2} \delta_{11} \xi \sum_{n=1}^{S} \int_{z_{n-1}^{+}}^{z_{n}^{-}}\left[w^{\prime \prime}(z, t)\right]^{2} d z \\
& +\frac{c L \xi}{2 \delta_{11}} \sum_{n=1}^{S} \int_{z_{n-1}^{+}}^{z_{n}^{-}}[\dot{P}(z, t)]^{2} d z
\end{aligned}
$$$$
\begin{aligned}
& -\sum_{n=1}^{S-1}\left(\rho_{n}-\rho_{n+1}\right)\left[\frac{\xi}{2} z_{n}^{-}-\zeta\left(L-z_{n}^{-}\right)\right]\left[\dot{P}\left(z_{n}^{-}, t\right)\right]^{2} \\
& +\frac{(\alpha+\zeta+\xi) \rho_{\max } L g}{2 \delta_{7}}+\frac{\xi \rho_{S} g L}{2 \delta_{13}} \\
& +\frac{(S-1)(\zeta+\xi) \rho_{\max } g L}{2 \delta_{10}}
\end{aligned}
$$

Using (B.7), (B.12) and (B.38), we can obtain

$$
\begin{aligned}
\dot{V}(t) \leq & -\sum_{n=1}^{S} \int_{z_{n-1}^{+}}^{z_{n}^{-}}\left[\alpha E I_{n}-2 L^{2}(\alpha+\zeta+\xi) \delta_{7} g \rho_{\max }\right. \\
& -\frac{8(S-1)(\zeta+\xi) \delta_{10} \rho_{\max } g L^{2}}{2}-\frac{8 \xi \delta_{13} \rho_{S} g L^{2}}{2} \\
& -\frac{3(\zeta+\xi)}{2} E I_{n}-2 L^{2} \alpha c \delta_{8}-c L^{2}(\zeta+\xi) \delta_{11} \\
& \left.-2 L^{2} \gamma \rho_{n} g \delta_{5}\right]\left[w^{\prime \prime}(z, t)\right]^{2} d z-\sum_{n=1}^{S} \int_{z_{n-1}^{+}}^{z_{n}^{-}}[\beta c \\
& -\frac{c \alpha}{2 \delta_{8}}-\alpha \rho_{n}\left(1+\frac{\delta_{6} L}{2}\right)-\frac{c L(\zeta+\xi)}{2 \delta_{11}}-\frac{L \zeta \delta_{9}}{2} \rho_{n} \\
& \left.-\frac{\zeta}{2} \rho_{n}-\frac{\beta \delta_{3} \rho_{n} g}{2}-\frac{\xi}{2} \rho_{n}\left(1+\delta_{12} L\right)\right][\dot{P}(z, t)]^{2} d z \\
& -\sum_{n=1}^{S-1}\left(\rho_{n}-\rho_{n+1}\right)\left[\frac{\xi}{2} z_{n}^{-}-\zeta\left(L-z_{n}^{-}\right)\right]\left[\dot{P}\left(z_{n}^{-}, t\right)\right]^{2} \\
& -\left(\frac{k}{2}-\frac{\xi \rho_{\max } L^{2}}{4 \delta_{12}}-\frac{\zeta \rho_{\max } L^{2}}{2 \delta_{9}}-\frac{\alpha \rho_{\max } L^{2}}{4 \delta_{6}}\right. \\
& \left.-\frac{\beta E I_{1}}{2 \delta_{4}}\right)[\dot{\theta}(t)]^{2}-\frac{\xi}{2} \sum_{n=1}^{S} \rho_{S} L[\dot{P}(L, t)]^{2} \\
& -\frac{E I_{1}}{2}\left(\zeta L-\beta \delta_{4}\right)\left[P^{\prime \prime}(0, t)\right]^{2}-\left(k_{\theta}-\frac{\gamma g \rho_{\max } L}{2 \delta_{5}}\right) \\
& \times\left[\dot{\theta}(t)+\theta_{e}(t)\right]^{2}-k_{u}\left[u_{a}(t)\right]^{2}-\frac{k}{2}\left[\theta_{e}(t)\right]^{2} \\
+ & \frac{(S-1)(\zeta+\xi) \rho_{\max } g L}{2 \delta_{10}}+\frac{\xi \rho_{S} g L}{2 \delta_{13}} \\
+ & \frac{(\alpha+\zeta+\xi) \rho_{\max } L g}{2 \delta_{7}}+\frac{\beta g L \rho_{\max }}{2 \delta_{3}} \\
&
\end{aligned}
$$

where

$$
\begin{aligned}
\varepsilon= & \frac{(S-1)(\zeta+\xi) \rho_{\max } g L}{2 \delta_{10}}+\frac{\xi \rho_{S} g L}{2 \delta_{13}} \\
& +\frac{(\alpha+\zeta+\xi) \rho_{\max } L g}{2 \delta_{7}}+\frac{\beta g L \rho_{\max }}{2 \delta_{3}}
\end{aligned}
$$

$\left.+\frac{\xi}{2} \rho_{n}\left(1+\delta_{12} L\right)+\frac{\zeta}{2} \rho_{n}+\frac{L \zeta \delta_{9}}{2} \rho_{n}\right][\dot{P}(z, t)]^{2} d z$

$-\frac{\xi}{2} \sum_{n=1}^{S} \rho_{S} L[\dot{P}(L, t)]^{2}+\left(\frac{\xi \rho_{\max } L^{2}}{4 \delta_{12}}+\frac{\zeta \rho_{\max } L^{2}}{2 \delta_{9}}\right.$

and the positive constants $\alpha, \beta, \gamma, \zeta, \xi, c, k, k_{u}, k_{\theta}$, $\delta_{1}-\delta_{13}$ are chosen under the following conditions.

$$
\left.+\frac{\alpha \rho_{\max } L^{2}}{4 \delta_{6}}\right)[\dot{\theta}(t)]^{2}-\frac{\zeta}{2} L E I_{1}\left[w^{\prime \prime}(0, t)\right]^{2}
$$

$\beta>\max \left\{\frac{2 L \rho_{\max }\left(2 L \alpha \delta_{1}+\zeta \delta_{2}\right)}{E I_{\min }}, \frac{\zeta}{\delta_{2}}+\frac{\alpha}{\delta_{1}}\right\}$ 
$\kappa_{n}=\alpha E I_{n}-2 L^{2}(\alpha+\zeta+\xi) \delta_{7} g \rho_{\max }-2 L^{2} \alpha c \delta_{8}$ $-\frac{8(S-1)(\zeta+\xi) \delta_{10} \rho_{\max } g L^{2}}{2}-2 L^{2} \gamma \rho_{n} g \delta_{5}$$$
-\frac{3(\zeta+\xi)}{2} E I_{n}-\frac{8 \xi \delta_{13} \rho_{S} g L^{2}}{2}
$$$$
-c L^{2}(\zeta+\xi) \delta_{11}>0, n \in\{1,2, \ldots, S\}
$$

$\nu_{n}=\beta c-\frac{c \alpha}{2 \delta_{8}}-\frac{\beta \delta_{3} \rho_{n} g}{2}-\frac{c L(\zeta+\xi)}{2 \delta_{11}}-\frac{L \zeta \delta_{9}}{2} \rho_{n}$ $-\alpha \rho_{n}\left(1+\frac{\delta_{6} L}{2}\right)-\frac{\xi}{2} \rho_{n}\left(1+\delta_{12} L\right)-\frac{\zeta}{2} \rho_{n}>0$, $n \in\{1,2, \ldots, S\}$ (B.43)

$$
\begin{aligned}
\chi_{1}= & \min \left\{\frac{2 \nu_{1}}{\beta \rho_{1}}, \ldots, \frac{2 \nu_{S}}{\beta \rho_{S}}\right\} \\
= & -\frac{\delta_{6} \alpha L}{\beta}-g \delta_{3}-\frac{L \zeta \delta_{9}}{\beta}-\frac{2 \alpha+\zeta+\xi\left(1+\delta_{12} L\right)}{\beta} \\
& -\frac{c L(\zeta+\xi)}{\beta \delta_{11} \rho_{\max }}-\frac{c \alpha}{\delta_{8} \beta \rho_{\max }} \\
\chi_{2}= & \min \left\{\frac{2 \kappa_{1}}{\beta E I_{1}}, \ldots, \frac{2 \kappa_{S}}{\beta E I_{S}}\right\} \\
= & \frac{2 \alpha}{\beta}-\frac{3(\zeta+\xi)}{\beta}-\frac{8(S-1)(\zeta+\xi) \delta_{10} \rho_{\max } g L^{2}}{\beta E I_{\max }} \\
& -\frac{4 L^{2} \delta_{7} g(\alpha+\zeta+\xi) \rho_{\max }}{\beta E I_{\max }}-\frac{2 c L^{2} \delta_{11}(\zeta+\xi)}{\beta E I_{\max }} \\
& -\frac{4 c L^{2} \delta_{8} \alpha}{\beta E I_{\max }}-\frac{4 L^{2} \rho_{n} g \gamma \delta_{5}}{\beta E I_{\max }}-\frac{8 \xi \delta_{13} \rho_{S} g L^{2}}{\beta E I_{\max }} \\
\sigma_{1}= & \frac{k}{2}-\frac{\rho_{\max } L^{2}}{2}\left(\frac{\zeta}{\delta_{9}}+\frac{2 \xi}{\delta_{12}}+\frac{\alpha}{2 \delta_{6}}\right)-\frac{\beta E I_{1}}{2 \delta_{4}}>0(\mathrm{~B} . \\
\sigma_{2}= & \frac{\xi}{2} z_{n}^{-}-\zeta\left(L-z_{n}^{-}\right)>0 \\
\sigma_{3}= & k_{\theta}-\frac{\gamma g \rho_{\max } L}{2 \delta_{5}}>0 \\
\sigma_{4}= & \zeta L-\beta \delta_{4}>0
\end{aligned}
$$

where $E I_{\max }$ is the maximum value of $E I_{n}, n \in\{1,2$, $\ldots, S\}$.

$\lambda_{3}=\min \left\{\chi_{1}, \chi_{2}, \frac{2 \sigma_{3}}{J_{A} \gamma}, 1, \frac{2 k_{u}}{m_{c}}\right\}>0$.

Combining (A.4) and (B.39), we finally obtain

$\dot{V}(t) \leq-\lambda V(t)+\varepsilon$,

where $\lambda=\lambda_{3} / \lambda_{2}>0$.

\section{Proof of Step 3}

Proof : Multiplying inequation (B.51) by $e^{\lambda t}$ yields $\frac{\partial}{\partial t}\left[V(t) e^{\lambda t}\right] \leq \varepsilon e^{\lambda t}$.

Integrating of the above inequality leads to

$V(t) e^{\lambda t}-V(0) \leq \frac{\varepsilon}{\lambda} e^{\lambda t}-\frac{\varepsilon}{\lambda}$ then we can obtain

$V(t) \leq\left[V(0)-\frac{\varepsilon}{\lambda}\right] e^{-\lambda t}+\frac{\varepsilon}{\lambda} \leq V(0) e^{-\lambda t}+\frac{\varepsilon}{\lambda} \in \mathscr{L}_{\infty}$

which suggests $V(t)$ is bounded. Using (16), (A.4) and (12), we obtain

$$
\begin{aligned}
\frac{\beta E I_{n}}{16 L}[w(z, t)]^{2} & \leq \sum_{n=1}^{S} \int_{z_{n-1}^{+}}^{z_{n}^{-}} \frac{\beta E I_{n}}{2}\left[w^{\prime \prime}(z, t)\right]^{2} d z \\
& \leq V_{I}(t) \leq V_{I}(t)+V_{I I}(t) \leq \frac{1}{\lambda_{1}} V(t) \in \mathscr{L}_{\infty}
\end{aligned}
$$

$\forall(z, t) \in\left[z_{n-1}^{+}, z_{n}^{-}\right] \times[0, \infty), n \in\{1,2, \ldots, S\}$. From (C.3) and (C.4), we have

$$
\begin{aligned}
|w(z, t)| & \leq \sqrt{\frac{16 L}{\lambda_{1} \beta E I_{n}}\left[V(0) e^{-\lambda t}+\frac{\varepsilon}{\lambda}\right]} \\
& \leq \sqrt{\frac{16 L}{\lambda_{1} \beta E I_{n}}\left[V(0)+\frac{\varepsilon}{\lambda}\right]}
\end{aligned}
$$

$\forall(z, t) \in\left[z_{n-1}^{+}, z_{n}^{-}\right] \times[0, \infty), n \in\{1,2, \ldots, S\}$. Further more, from (C.5), we have

$\lim _{t \rightarrow \infty}|w(z, t)| \leq \sqrt{\frac{16 L \varepsilon}{\lambda \lambda_{1} \beta E I_{n}}}$

$\forall(z, t) \in\left[z_{n-1}^{+}, z_{n}^{-}\right] \times[0, \infty), n \in\{1,2, \ldots, S\}$. Using (17) and (A.4), we have

$\frac{k}{2}\left[\theta_{e}(t)\right]^{2} \leq V_{I I}(t) \leq V_{I}(t)+V_{I I}(t) \leq \frac{1}{\lambda_{1}} V(t) \in \mathscr{L}_{\infty}$

From (C.3) and (C.7), we have

$\left|\theta_{e}(t)\right| \leq \sqrt{\frac{2}{k \lambda_{1}}\left[V(0) e^{-\lambda t}+\frac{\varepsilon}{\lambda}\right]} \leq \sqrt{\frac{2}{k \lambda_{1}}\left[V(0)+\frac{\varepsilon}{\lambda}\right]}$

Further more, from (C.8), we have

$\lim _{t \rightarrow \infty}\left|\theta_{e}(t)\right| \leq \sqrt{\frac{2 \varepsilon}{k \lambda \lambda_{1}}}, \quad \forall t \in[0, \infty)$. 